Authors' reply: It is of course impossible to disagree with Dr Van Houdenhove's statement that it is important to listen to the patient's life history. We have been doing this for many years. Therefore, the reason that he finds PTSD in so many of his patients with CFS and we do not is not our lack of listening. From previous presentations by this group from Belgium we know that they recruit patients with CFS with higher psychopathology scores than we do in our centre and than is usually found in other CFS cohorts. Hence it is understandable that they find higher rates of psychiatric comorbidity. To date there are no controlled studies indicating that a history of abuse is a characteristic of many patients with CFS.

J. Prins Medical Psychology, University Medical Centre St Radboud, 6500 HB Nijmegen,

The Netherlands. E-mail: j.prins@mps.umcn.nl

G. Bleijenberg Medical Psychology, University Medical Centre St Radboud, Nijmegen,

The Netherlands

\section{Not all women fancy single-sex wards}

Mezey et al (2005) discuss staff and patient perceptions of the safety of women in mixed-sex and single-sex medium secure units. In their conclusion, the authors ask 'how much choice women should be allowed to exert over the type of setting [single-sex or mixed-sex wards] where they receive treatment'. On the basis of our own survey we can provide tentative answers to the related question of whether they want a choice, and, if so, what they would choose.

As part of a much larger study (in preparation), we conducted a survey of 50 inpatients (31 women) who were admitted to four mixed-sex acute psychiatric wards. We explored their ward milieu preferences: single-sex $v$. mixed-sex wards. The mean age of the sample was 39.5 years (s.d.=13.49) and the common diagnostic categories were schizophrenia $(45 \%)$ and affective disorders (20\%). Although 24 patients $(48 \%)$ stated that they would like a choice in the type of ward they were admitted to, only $16(32 \%)$ preferred single-sex wards to mixed-sex wards. A gender-wise analysis further showed that of the 16 who preferred single-sex wards, only $4(25 \%)$ were women. The remainder of the female sample (27 out of 31 ) preferred mixed-sex wards. Of course, the reasons given for this choice were varied and complex.

Hence, our findings do not support the widely held belief that women often prefer single-sex wards. In view of the small sample size of our study, more work needs to be done on this subject before definitive conclusions are drawn.

Mezey, G., Hassell, Y. \& Bartlett, A. (2005) Safety of women in mixed-sex and single-sex medium secure units: staff and patient perceptions. British Journal of Psychiatry, 187, 579-582.

V. Pemmaraju Queen Elizabeth Psychiatric Hospital, Birmingham BI5 2QZ, UK.

E-mail: vinuthna@doctors.org.uk

S. George, F. Oyebode Queen Elizabeth

Psychiatric Hospital, Birmingham, UK

Psychiatric hospitals operate at three levels (Stokes, 1994) - 'what we say we do', 'what we really believe we are doing' and 'what is actually going on'. Most are unconscious of this third level. Mezey et al (2005) rightly remind us that the further development of single-sex secure units for women may not be justified on the grounds of safety alone, but such a narrow focus has never been the principal driving force for developing gender-sensitive and single-sex secure units, which has massive ramifications for the wider hospital organisation.

Ramsay et al (2001) broadly described the issues relating to women and psychiatry and Kennedy (2001) argued for a proper focus on the needs of male patients. Both perspectives reinforce the need for genderinformed practice. While developing different services for male and female forensic patients, it is worth considering that one tenet of feminism is that no person should be discriminated against on the grounds of gender. Although men and women are not the same, Adshead (2004) argues that, in terms of human needs and human rights, male and female patients are more alike than they are different and that differences should not be the basis for abusive or discriminatory practice.

Although not emphasised sufficiently in the literature, particular issues are commonly encountered by both male and female in-patient forensic populations. These include the emotional and behavioural impact of bringing together a number of patients who have high levels of comorbidity, personality disorder and complex post-traumatic stress syndromes (Bercu, 2001). However, models of care for male patients (the majority population in mixed forensic units) fail to address the relational and other specific needs of women, their marginalisation and the impact of their experience of victimisation. Gender-informed forensic services therefore need to be equipped and enabled to provide appropriate levels of care and interventions, in suitable accommodation, for individuals with severe, complex clinical presentations and who present significant risks to themselves and others.

Our position is not that gender adequately differentiates between men's and women's needs but that in the absence of more sophisticated frameworks, their needs are such that, for the foreseeable future, service planning must be based on the assumption that women forensic patients are sufficiently different from their male counterparts that their needs should be provided for separately.

\section{Declaration of interest}

None. This is not a representative view of Nottinghamshire Healthcare NHS Trust, Nottingham City Primary Care Trust or The University of Manchester.

Adshead, G. (2004) More alike than different. In Working Therapeutically with Women in Secure Mental Health Settings (eds N. Jeffcote \& T.Watson). London: Jessica Kingsley.

Bercu, S. (200I) Experience of a women's psychiatric ward in London (abstract). Abstracts of the 26th International Congress on Law and Mental Health. Canada: Cheneliere/McGraw-Hill. http://www.ialmh.org/ Montreal2001/Sessions/womens_corrections.htm

Kennedy, H. (200I) Do men need special services? Advances in Psychiatric Treatment, 7, 93-99.

Mezey, G., Hassell, Y. \& Bartlett, A. (2005) Safety of women in mixed-sex and single-sex medium secure units: staff and patient perceptions. British Journal of Psychiatry, 187, 579-582.

Ramsay, R., Welch, C. \& Youard, E. (200I) Needs of women patients with mental illness. Advances in Psychiatric Treatment, 7, 85-92.

Stokes, J. (1994) Institutional chaos and personal stress. In The Unconscious at Work (eds A. Obholzer \& V. Z. Roberts). London: Routledge.

R. F. Travers National High Secure Women's Services, Nottinghamshire Healthcare NHS Trust, Rampton Hospital, Retford, East Notts DN22 OPD, UK. E-mail: raymond.travers@nottshc.nhs.uk

P. Holdsworth Nottingham City Primary Care Trust, Nottingham, UK

D. Edge Faculty of Medical and Human Sciences, The University of Manchester, Manchester, UK 